\title{
Historia de la Atención Primaria de Salud en Perú: entendiendo su camino y perspectivas actuales
}

\author{
History of Primary Health Care in Peru: understanding its path and current perspectives \\ Luis Fernando Llanos Zavalaga ${ }^{1, a, b, c}$, Daniella Arenas Siles ${ }^{2,3, a}$, Bryan Valcarcel ${ }^{2, a}$, \\ Oscar Huapaya Huertas 2,a,d
}

\section{RESUMEN}

El desarrollo de la atención primaria de salud en el Perú ha logrado importantes hitos en su historia. Los fundamentos sociales que dieron forma a los servicios de salud en el siglo anterior pueden explicarse por eventos durante la época colonial. Además, después de la Declaración de Alma-Ata en 1978, Perú enfrentó varios obstáculos que polarizaron aún más a la población de diferentes estratos económicos. Sin embargo, el Ministerio de Salud, junto con la cooperación internacional, comenzó a desarrollar programas de salud con el objetivo de brindar atención primaria a la población, con resultados fundamentales que ayudaron a unificar a la población y a estar un paso más cerca de lograr una cobertura de atención médica universal. Por lo tanto, en este documento, describimos los eventos que conducen al sistema de salud actual del Perú, los logros de los servicios de Atención Primaria de Salud y el uso de dispositivos tecnológicos para mejorar la salud pública.

PALABRAS CLAVE: Salud pública; atención primaria de salud; cooperación internacional. (Fuente: DeCS BIREME).

\section{SUMMARY}

The development of primary health care in Peru has achieved important milestones in its history. The social foundations that were formed in the health services in the previous century can be explained by events during the colonial era. Furthermore, after the Declaration of Alma-Ata in 1978, Peru faced several obstacles that further polarized the population from different economic strata. However, the Ministry of Health, together with international cooperation, began to develop health programs with the objective of providing primary care to the population, with the fundamental results that will help to unify the population and be one-step closer to achieving universal health care coverage. Therefore, in this document, we describe the events leading to Peru's current health system, the achievements of Primary Health Care services, and the use of technological devices to improve public health.

KEYWORDS: Public health, primary health care, international cooperation. (Source: MeSH NLM).

1 Universidad Peruana Cayetano Heredia. Lima, Perú.

2 Universidad Científica del Sur. Lima, Perú.

3 Hospital Guillermo Almenara Irigoyen, EsSalud. Lima, Perú.

a Médico Cirujano;

b Especialista en Administración en Salud;

c Maestría en Políticas, Planificación y Financiamiento en Salud,

d Maestría en Informática Biomédica, 


\section{INTRODUCCIÓN}

La historia del desarrollo del sistema de atención médica en Perú es digna de comentar, desde los cambios espurios y drásticos que surgieron en la época colonial hasta la superficial gestión que condujo a situaciones precarias de la salud en las últimas décadas (1). Estos eventos se fusionaron y culminaron con un sistema de salud segregado, con marcadas diferencias entre el estatus socioeconómico y la condición étnica (2). Por lo tanto, Perú enfrentó un desafío oneroso cuando la declaración de Alma-Ata estableció la base para la reforma de salud global, con la participación no solo del estado peruano o de los profesionales de la salud, sino la inclusión y la participación activa de la comunidad en el manejo de su propia salud.

La Organización Mundial de la Salud (OMS) adoptó un nuevo concepto para brindar salud con la declaración de Alma-Ata en 1978, durante la Conferencia Internacional sobre Atención Primaria de Salud. Este evento cosmopolita sentó las bases de la Atención Primaria de Salud (APS), articulada en varios puntos. Dos de ellos son de importante consideración. El primero es el empoderamiento de la salud comunitaria e individual como motor para prosperar la atención médica. Esta característica les dio el derecho y el deber de planificar e implementar sistemas de salud. El segundo punto es un esfuerzo ecléctico de instituciones nacionales e internacionales, con la intención de revertir las desigualdades en salud en los países en desarrollo.

Sin embargo, los académicos acusaron que estas estrategias eran demasiado amplias y holísticas, sin medios pragmáticos para llevarlas a cabo en todos los países, especialmente en los que se encontraban en vías de desarrollo (3). Dos puntos de vista polarizaron a la comunidad académica hacia los países menos desarrollados; el primero argumenta que el deterioro de la salud es un problema social y económico que podría revertirse con la respuesta política, mientras que el segundo afirma que estos países necesitaban la tecnología adecuada para contrarrestar sus obstáculos. En ese momento, para lograr el deseo "Salud para todos" en Perú, el Estado primero tendría que reducir las disparidades socioeconómicas en la población, promover una colaboración eficiente entre los sectores gubernamentales, unificar el sistema de salud fragmentado y hacerlo sostenible (4).

Esta tarea desarrolló algunos programas y reformas importantes que contribuyen a la mejora de la salud, aunque sin alcanzar el concepto de APS de la Conferencia Internacional. Por lo tanto, en esta revisión, nuestro objetivo es relatar los eventos históricos del sistema de salud peruano que condujeron al estado actual de la APS en Perú y describir las intervenciones de salud pública pasadas y actuales, con énfasis en la tecnología de salud.

\section{Historia de los seguros de salud peruanos}

En breve, el primer seguro de salud, el Servicio de Salud de la Policía, data de 1924, el cubre las fuerzas armadas. Sin embargo, en 1936, el gobierno de Perú creó la Seguridad Social de los Trabajadores Nacionales, el primer intento de cobertura nacional de salud. Este seguro proporcionaba cobertura de salud a los trabajadores y se sostenía con una cuota del salario de ellos. En los años siguientes, el gobierno reforma el concepto principal de este seguro al extender la cobertura de la población creando el Instituto Peruano de Seguridad Social (IPSS), que posteriormente permitió la creación del actual Seguro de Salud Social (EsSalud), en 1999.

El Ministerio de Salud (MINSA), luego de los esfuerzos iniciales para promover el seguro público a través del Seguro Escolar Gratuito (SEG) y el Seguro Materno Infantil (SMI), creó el Seguro Integral de Salud (SIS) en 2001, capaz de proporcionar cobertura en dos formas. La primera mediante el esquema subsidiado, diseñado para personas en condiciones pobres y extremadamente pobres. La segunda forma es mediante el esquema semi subsidiado, donde las personas abonan un pequeño monto. En cuanto al sector privado, se creó en el año 1991, en respuesta a la hegemonía del seguro de salud por parte del gobierno. Estos hitos evidencian la división actual del sistema de salud pública (EsSalud, SIS, PNP y FFAA) y el sector privado, con un producto de 5 seguros de salud diferentes (5).

\section{Desarrollo de la salud antes de la declaración de Alma-Ata}

Antes de que los españoles conquistaran el imperio inca, el suministro de alimentos, el ganado, las fuentes de agua limpia y la eliminación de desechos no eran un problema crítico para los habitantes oriundos. Con la fundación de Lima en 1535, se produjeron varias migraciones, incluidas las colonias españolas y esclavos africanos, lo que condujo a un rápido aumento de la población, escasez de alimentos y acumulación de desechos, lo que comprometió el estado de salud 
de todos los habitantes (1). Como respuesta, se construyeron hospitales para proporcionar atención sanitaria, sin embargo, con una distinción sólida entre los grupos étnicos. Así, el hospital San Andrés era para los españoles, el hospital Santa Ana para los indígenas y el hospital San Bartolomé para los esclavos africanos $(2,6)$. Aunque no está claro si estos hospitales sirvieron solo como instituciones comprometidas con la salud o si su objetivo principal era imponer la superioridad de los gobernadores españoles y aprovechar las ventajas económicas mediante la recaudación de ingresos de la comunidad $(2,7)$; la Corona española intentó regular el servicio de salud en estos hospitales y dentro de la comunidad (8).

En el año 1570, La Corona implementó sus propias medidas para regular las prácticas de salud a través de una institución llamada Protomedicato, creada en 1442. La misión del Protomedicato era evaluar la práctica correcta de la medicina, estudiar y clasificar las hierbas medicinales e informar a La Corona sobre asuntos relacionados a la salud. En cuanto a la práctica de la salud, el Protomedicato certificó que una persona estaba calificada para brindar servicios médicos en forma de 5 categorías: Doctor en Medicina, cirujano latino, cirujano romántico, flebotomista o farmacéutico (8). Al principio, solo los españoles y sus descendientes podían postularse para obtener la primera categoría; sin embargo, con el aumento de la población y la llegada de enfermedades europeas, la escasez de la oferto hizo que los habitantes interesados puedan practicar medicina; aunque nunca hubo una legislación que respalde esta acción $(1,8)$.

Durante la etapa del Protomedicato (casi 30 años) hasta la independencia peruana en 1821 , La Corona dictó varias regulaciones para la prestación de servicios de salud. Por ejemplo, la creación de escuelas de medicina y de políticas de salud (8). No obstante, la nueva e incipiente administración de la República condujo a decisiones cuestionables sobre el futuro del país, con 8 cambios en la constitución política nacional en menos de 50 años (9). El sector de salud reflejó esta inestabilidad: la administración hospitalaria no aseguró condiciones saludables para atender a sus pacientes y los médicos internacionales criticaron a sus pares peruanos por ser recalcitrantes a las nuevas modalidades de tratamiento (10).

Casi cien años después de la independencia, en 1933, un médico llamado Manuel Núñez Butrón, inició una doctrina en Puno llamada "Rijcharismo", la cual incitaba a la población a "despertar" y ser partícipes de su propia salud, mejorándola a través de adecuados estilos de vida. El objetivo de este movimiento era educar, proporcionar conocimiento de la salud a la población indígena de Puno y esforzarse por fusionar la cultura indígena con la práctica de la cultura occidental a través de dos acciones principales. La primera fue realizar sesiones grupales para promover la participación comunitaria activa en temas como la higiene y la importancia de la vacunación para prevenir enfermedades (11). La segunda acción fue la difusión de conceptos preventivos de salud a través de la revista "Runna Soncco", traducida como "corazón de indio", que incluía una mayor variedad de temas sanitarios que las sesiones comunitarias. A pesar de su tiempo efímero, hasta 1948, este movimiento es considerado un proyecto pionero y a escala para la futura Declaración de Alma-Ata, 30 años después (12).

\section{Desarrollos de APS durante la declaración de Alma-Ata y años posteriores}

El director de la OMS, Halfdan Mahler, con la ayuda de David Tejada - un médico peruano -, planeó, articuló y presentó la Declaración de Alma-Ata en el año 1978. Desde entonces, el concepto de APS cambió hacia una prestación de servicios de salud colaborativa entre la comunidad y los trabajadores de salud $(3,10)$. Sin embargo, a pesar de las promesas que conlleva este concepto, en el Perú estaba a punto de comenzar una crisis política, junto con una subversión sociopolítica impulsada por un motivo de desigualdad socioeconómica, que destrozaría la inmersión de este concepto en la población. Esta revolución, llamada "Sendero Luminoso", dirigida por Abimael Guzmán, causó graves daños, tanto sociales como materiales (13). Este acontecimiento dejó un asesinato masivo de 69000 personas y, en las consecuencias sociales, el daño colateral dejó a la población refractaria a la ayuda del Gobierno $(13,14)$.

Después del final del período inestable del terrorismo, se llevaron a cabo varios programas relacionados con la APS, tanto de cooperación nacional, internacional bilateral como multilateral. No obstante, tres de ellos, de cada categoría, se consideran proyectos fundamentales en el proceso de implementación de APS, por su alta cobertura demográfica, alto apoyo financiero y el impacto en la salud pública (15). 


\section{Programa Administrativo Compartido (PAC) y el}

Comité de Administración de Salud Local (CLAS)

Como parte de la reforma de salud y el proceso de descentralización, el Estado peruano y el Ministerio de Salud (MINSA) crearon el Programa Administrativo Compartido (PAC) y el Comité de Administración de Salud Local (CLAS), establecidos en el Decreto Supremo No 01-94-SA en el año 1994. El PAC sirvió para promover y articular la atención médica de la comunidad a través del CLAS, definido como una persona jurídica auto gestionada sin fines de lucro y conformado por tres ejes: representantes de la comunidad, representantes del Estado y el director o el establecimiento de salud local. Este movimiento comenzó a funcionar, bajo la administración del MINSA, a la par con otros centros de atención primaria de salud en el mismo entorno regional.

Sin embargo, el CLAS incorpora y une a la comunidad junto a los profesionales de la salud, referido como cogestión, para aumentar la cobertura de salud, adaptar las necesidades de salud específicas, disminuir el costo de la salud y mejorar la prestación de atención médica (16). A nivel local, el balance del CLAS fue favorable, los pacientes informaron una mayor tasa de satisfacción, un menor tiempo de espera para la consulta médica y una exoneración de pagos más flexibles a las personas con escasos recursos económicos (17).

Como resultado, en el año 2008 la Ley N $N^{\circ} 29124$ determinó la iniciativa CLAS como modelo para las instalaciones de APS bajo la jurisdicción del MINSA, para proporcionar servicios de salud equitativos a la población (18). Posteriormente, se desarrollan normativas que no tienen una implementación sostenible con tres abordajes semejantes: las Redes Integradas de Atención Primaria en Salud (RIAPS) creado con Decreto Legislativo $\mathrm{N}^{\circ} 1166$ que establece en diciembre de 2013 que es el conjunto de IPRESS públicas, privadas o mixtas que se articulan funcionalmente a través de acuerdos de carácter institucional o contractual para prestar servicios de promoción, prevención, recuperación y rehabilitación de la salud a una población definida y ubicada en un espacio geográfico determinado. Asimismo, coordina con las diferentes entidades públicas y privadas que actúan en su ámbito geográfico para el abordaje de los determinantes de la salud.

En diciembre de 2016, se emite el Decreto Legislativo que optimiza el funcionamiento y los servicios del sector Salud que señala que el MINSA, como Autoridad Sanitaria Nacional, establece el alcance, conformación, instrumentos y mecanismos necesarios de relación funcional entre IPRESS y redes de IPRESS (centros y puestos), así como las disposiciones complementarias y conexas para su implementación; además, este decreto deroga el Decreto Legislativo $\mathrm{N}^{\circ} 1166$.

Posteriormente en noviembre del 2018 se emite la Ley que establece la conformación y el funcionamiento de las Redes Integradas de Salud (RIS). Estas son definidas como un "Conjunto de organizaciones que presta, o hace los arreglos institucionales para prestar una cartera de atención de salud equitativa e integral a una población definida, a través de la articulación, coordinación y complementación, y que rinde cuentas por los resultados sanitarios y administrativos y por el estado de salud de la población a la que sirve".

Finalmente, en mayo del 2020 se emite el Decreto Supremo No 019-2020-SA aprobando el Reglamento de la Ley $\mathrm{N}^{\circ} 30885$. Se espera que este sea una primer paso en el rediseño del sistema sanitario nacional hacia un sistema articulado e integrado, en lugar de un sistema segmentado y fragmentado.

\section{Cooperación bilateral}

El Gobierno del Perú, junto con la Agencia de los Estados Unidos para el Desarrollo Internacional (USAID) creó el programa "Proyecto 2000" en 1994. El objetivo fue reducir la mortalidad materna y perinatal a través de tres temas específicos: asegurar recursos humanos y materiales, aumentar la aceptabilidad de la comunidad hacia los centros de atención médica al disminuir las brechas interculturales, y mejorar la calidad de atención de salud materna e infantil mediante el diseño de indicadores de salud específicos (19). El programa se efectuó en 12 departamentos (Ancash, Andahuaylas, Ayacucho, Huancavelica, Ica, La Libertad, Lima Este, Moquegua, Puno, San Martín, Tacna y Ucayali), con un presupuesto de 60 millones de dólares. Como resultado, El Proyecto 2000 redujo un $25 \%$ la tasa general de mortalidad maternal e incrementó 4 veces las probabilidades de supervivencia de un recién nacido, comparado con los datos previos a la intervención. Además, los recursos humanos y materiales, la calidad de la atención médica y la aceptación general de las instituciones de salud tuvieron resultados favorables en los departamentos seleccionados. No obstante, durante la duración del proyecto, hubo varios cambios en los coordinadores de 
las instituciones de salud médica en la comunidad, lo que retrasó la adquisición de recursos materiales; por lo tanto, una de las lecciones aprendidas fue designar a un coordinador de recursos inamovible, el cual no sea afectado por los cambios de personal efectuados por el MINSA $(19,20)$.

\section{Cooperación multilateral}

En cuanto a la cooperación multilateral, varias agencias diseñaron y ayudaron a la población mediante programas de APS, sin embargo, uno de ellos es aclamado como el más grande y ambicioso de la década (15). Esta intervención fue el "Proyecto Básico de Salud y Nutrición (PNSB)", desarrollado en 4 departamentos del Perú: Piura, Cusco, Cajamarca y Lima; y un total de 2150000 personas. El proyecto, financiado por el Banco Mundial con 34 millones de dólares, tenía como objetivo evaluar y mejorar la condición de salud y nutrición de las mujeres en edad fértil y de los niños menores de 3 años (21). Al final del PNSB en el año 2000, el cumplimiento global de seis de sus objetivos específicos excedió el 100\%, y superó el $95 \%$ para los dos restantes. Además, con esta experiencia, el MINSA identificó que trabajar con agentes comunitarios y organizaciones sin fines de lucro especializadas en temas de promoción de la salud -asociado a un plan de gestión sólido previo a la intervención-, son los dos puntos indispensables para lograr un impacto en la sociedad a través de un programa de salud comunitario (22).

\section{Cobertura universal de salud}

El Gobierno peruano, luego de los proyectos de salud pública, comenzó una campaña ambiciosa para brindar cobertura de salud a toda la población. Por lo tanto, el Gobierno creó la Cobertura Universal de Salud (CUS) y promovió el Modelo de Atención Integral de Salud (MAIS) en el 2003 (23). Durante los primeros años de esta reforma, el programa no tuvo éxito en la comunidad. Por ello, su objetivo principal cambió y se centralizó en la familia y la comunidad, adoptando el nombre de Modelo de Atención Integral de Salud Basada en la Familia y la Comunidad (MAIS-BFC) (24). Para el año 2013, el gobierno confirmó 23 leyes, con la ambición de obtener una CUS definitiva y proporcionar APS a la población en los años siguientes (25).

A pesar de los auspiciosos resultados en salud pública, el actual sistema de salud permanece con una estructura fragmentada (26). Este sistema es producto de acciones administrativas independientes de diferentes entidades, recalcitrantes a establecer un sistema unificado y una administración exigua por parte del Gobierno a través de todos sus periodos políticos (27). Sin embargo, el camino hacia la cobertura universal es prometedor. A pesar de obtener un $100 \%$ de cobertura, el último censo nacional en 2017 indicó que el $75.5 \%$ de la población tenía algún tipo de seguro de salud, un aumento de aproximadamente el 30\% en contraste con el censo del 2007 (28).

Además, algunos indicadores importantes consiguieron superar la meta establecida por los Objetivos de Desarrollo Sostenible (ODS). Estos son la mortalidad en niños menores de 5 años y recién nacidos, con una tasa de 18 y 10 muertes por cada 1000 recién nacidos; por debajo del umbral de 25 y 12 muertes por cada 1000 recién nacidos de los ODS, respectivamente. En cambio, la mortalidad materna, con una tasa de 93 muertes por 100000 recién nacidos, aún no alcanza la tasa de 70 muertes. Sin embargo, esta tasa continúa disminuyendo. En el período 19901996 fue de 265 por cada 100000 recién nacidos, observándose una reducción de $64,9 \%$ comparado con la tasa materna actual (29).

Otro factor importante de esta reforma es la satisfacción de la población con respecto a la prestación de servicios de salud, donde la última encuesta nacional identificó una aprobación del 73.9\% (30). Este porcentaje expresa una aceptación del sistema de salud en la mayoría de la población, que es el resultado de la inclusión de personas de diferentes orígenes étnicos, económicos y culturales.

\section{Dispositivos tecnológicos en la atención primaria de la salud}

Una estrategia para lograr la cobertura universal de salud es la implementación de tecnología de salud, definida por la OMS como el uso de conocimiento sistemático y organizado en forma de dispositivos, medicamentos, vacunas, procedimientos y sistemas desarrollados (31). En el año 2005, el primer intento legal de este programa fue instigado por el Plan Nacional de Telesalud, donde se efectuaron subsiguientes regulaciones políticas con el objetivo de consolidar la entrega de APS (32). El uso principal de este programa fue a través de dispositivos tecnológicos, como teléfonos inteligentes o computadoras, permitiendo comunicación entre establecimientos de salud en áreas de difícil acceso; proporcionando a los trabajadores de salud herramientas para visualizar 
imágenes radiológicas, leer e informar los resultados de laboratorio; o educando a la comunidad en la promoción y prevención de la salud (33-39). Por ejemplo, en Amazonas se implementó el Enlace Hispano Americano de Salud, el cual transmite mensajes de voz y de correo electrónico. Antes de este programa, la comunicación entre el personal de salud de la región era agotadora; sin embargo, después de nueve meses de la implementación de este programa, se pudo conseguir el $100 \%$ de comunicación sobre la evacuación de emergencia y $93 \%$ de consultas con otro profesional de salud para discutir un caso clínico y decidir su tratamiento - previamente el porcentaje era de $40 \%$ (evacuación de emergencia) y $6 \%$ (discusión de caso) para ambas situaciones (39).

Con respecto a los médicos, un sistema destinado a informar los resultados del laboratorio de las pruebas de tuberculosis disminuyó el retraso de la prueba de susceptibilidad a los medicamentos y los resultados del cultivo en $43 \%$ y $32 \%$, respectivamente; lo que contribuyó a una rápida decisión clínica, evidenciada en una reducción de 16 días a la conversión del cultivo (34). Otro ejemplo fue la creación de una plataforma de tele colposcopía utilizando el colposcopio de bolsillo como dispositivo, que resultó ser comparable a la prueba de colposcopia convencional con el espacio de mejora en la configuración de imágenes y archivos electrónicos (38).

En lo que respeta a la comunidad, ejemplos de implementaciones exitosas son las proporcionadas por Young et al. (35), y Rubinstein et al. (36). En el primero, los autores utilizaron las redes sociales para educar sobre prácticas sexuales seguras y alentar las pruebas de detección del VIH en hombres que tienen sexo con hombres (HSH). El resultado principal fue un aumento de 2,6 veces de HSH acudiendo a las pruebas de VIH. En el segundo estudio, utilizaron teléfonos móviles para promover prácticas de estilo de vida saludable en entornos urbanos con bajos recursos y lograr reducir la circunferencia de la cintura y aumentar el consumo de frutas en el área. Como parte de la evaluación de impacto del Programa Nacional de Alimentación Escolar Qali Warma, se utilizó fotografías de cavidad oral para evaluar problemas de caries dental y un aplicativo en celular para evaluar problemas de audición en población escolar del país.

\section{CONCLUSIÓN}

El desarrollo del sistema de salud en Perú está marcado con un manejo irregular que resultó en una estructura fragmentada y segregada. Después de la Declaración de Alma-Ata, Perú enfrentó un período de terrorismo que redujo las oportunidades de mejora en relación a la cobertura de salud y la inclusión de APS en la población. Sin embargo, con el fin del terrorismo, el MINSA junto con el apoyo de instituciones internacional diseñó y llevó a cabo programas de APS que ayudaron a congregar y conciliar la relación fragmentada entre el gobierno peruano y la población en la década de los 90. De este modo, el MAIS -BFC busca una cobertura de salud del $100 \%$ en todos los sectores demográficos, económicos y sociales, con un enfoque en salud primaria, necesidades especiales de edad y requisitos específicos de la comunidad. Además, con el reconocimiento de la implementación de nuevas tecnologías en todas las áreas del mundo para mejorar la APS en la nueva Declaración de Astaná (40), creemos que la reforma de la atención de telesalud en Perú debería ser una de las fuerzas impulsoras para necesita una cobertura sanitaria universal. Sin embargo, para lograr este objetivo, el Gobierno debe unificar los diferentes sectores de atención médica y prosperar para una administración transparente de atención médica.

\section{Declaración de financiamiento y de conflictos de intereses:}

Financiado por los autores. Declaran no tener conflictos de interés.

\section{Correspondencia:}

Luis Fernando Llanos Zavalaga

Dirección: Calle Las Lilas 435 - Urb. San Eugenio. Lince

Teléfono: (511)-999660680

Correo electrónico: luis.1lanos@upch.pe

\section{REFERENCIAS BIBLIOGRÁFICAS}

1. Rivasplata PE. Salud pública impulsada por el Cabildo de Lima durante la Colonia. Anu colomb histo soc cult. 2014; 41(1):35.

2. Peralta KKd. The Nature of Colonial Bodies: Public Health in Lima, Peru, 1535-1635. Indiana: University of Notre Dame; 2015.

3. Cueto M. The origins of primary health care and selective primary health care. Am J Public Health. 2004; 94(11):1864-74.

4. Bryant JH, Richmond JB. Alma-Ata and Primary Health Care: An Evolving Story. In: Quah SR, editor. International Encyclopedia of Public Health (Second Edition). Oxford: Academic Press; 2017. p. 83-102. 
5. Seinfeld J, Montañez V, Besich N. The Health Insurance System in Peru: Towards a Universal Health Insurance. Washington DC: Global Development Network; 2013.

6. Salaverry O, Cárdenas-Rojas D. Establecimientos asistenciales del sector salud, Perú 2009. Rev Peru Med Exp Salud Publica. 2009; 26:264-7.

7. Ramos G. Indian hospitals and government in the colonial Andes. Med Hist. 2013; 57(2):186-205.

8. Zavala A. El Protomedicato en el Perú. Acta Médica Peruana. 2010; 27:151-7.

9. Romaní CB. Cuatrocientos Años de la Salud Pública en el Perú, 1533-1933. 1ra ed. Lima: UNMSM; 2004.

10. Cueto M, Lossio J, Pasco C. El rastro de la salud en el Perú. 1ra ed. Lima: IEP Ediciones; 2009.

11. Salinas D. Manuel Núñez Butrón: pionero de la atención primaria en el Mundo. Revista médica de Chile. 2014; 142(12):1612-3.

12. Arroyo-Hernandez CH. Runa soncco: Manuel Nunez Butron and his health education project. Rev Peru Med Exp Salud Publica. 2013; 30(2):336-9.

13. Bowyer T. Popular participation and the state: democratizing the health sector in rural Peru. Int J Health Plann Manage. 2004; 19(2):131-61.

14. Malone E. The shining path of Peru: Defeated or alive? Washington DC: Georgetown University; 2010.

15. Arósquipa C, Pedroza J, Cosentino C, Pardo K. La ayuda oficial al desarrollo en salud en el Perú. Rev Peru Med Exp Salud Publica. 2007; 24:163-78.

16. Ministerio de Salud. Local Health Administration Committees (CLAS): Organization and management model - The local health program. Lima: Ministerio de Salud; 1996.

17. Cortez R. Equidad y Calidad de los Servicios de Salud: El Caso de los CLAS. Lima: Universidad del Pacífico, 1998.

18. Ministerio de Salud. Cogestión y Participación Ciudadana para el primer nivel de atención en los establecimientos de salud del Ministerio de Salud y de las Regiones. Ley 29124 y Reglamento D.S. 017 2008 - SA. Lima: Ministerio de Salud; 2008.

19. Velásquez A, Benavides B. Estudio Comparativo de la Calidad y Uso de Servicios de Salud MaternoPerinatal: 1997 - 2002. Lima: Ministerio de Salud/ USAID; 2003.

20. Seclen-Palacín JA, Jacoby ME, Benavides CB, et al. Efectos de un programa de mejoramiento de la calidad en servicios materno perinatales en el Perú: la experiencia del proyecto 2000. Revista Brasileira de Saúde Materno Infantil. 2003; 3:421-38.

21. Ministerio de Salud. Proyecto Salud y Nutrición Básica: Hacia un Nuevo Modelo de Atención. Lima: Ministerio de Salud; 1997.

22. Ministerio de Salud. Proyecto Salud y Nutrición Básica: Informe Final. Lima: Ministerio de Salud;
1999.

23. Ministerio de Salud. Resolución Ministerial № 7292003-SA/DM. Lima: Ministerio de Salud; 2003.

24. Ministerio de Salud. Modelo de Atención Integral de Salud basado en Familia y Comunidad. Lima: Ministerio de Salud; 2011.

25. Solari L. Reforma de salud en el Perú: apuntando hacia la cobertura universal. Rev Peru Med Exp Salud Publica. 2014; 31:623-5.

26. Lazo-Gonzales O, Alcalde-Rabanal J, EspinosaHenao MO. El sistema de salud en Perú. Situación y desafíos. Lima: Colegio Médico del Perú; 2016.

27. Maguiña C, Galán-Rodas E. Situación de la salud en el Perú: la agenda pendiente. Rev Peru Med Exp Salud Publica. 201; 28:569-70.

28. Instituto Nacional de Estadística e Informática. Perú: Perfil Sociodemográfico. Lima: Instituto Nacional de Estadística e Informática; 2017.

29. Instituto Nacional de Estadística e Informática. Encuesta Demográfica y de Salud Familiar (ENDES). Lima: Instituto Nacional de Estadística e Informática; 2017.

30. Instituto Nacional de Estadística e Informática. Encuesta Nacional de Satisfacción de Usuarios en Salud 2016. Lima: Instituto Nacional de Estadística e Informática; 2016.

31. World Health Organization. Sixtieth World Health Assembly: Health Technologies. Geneva: World Health Organization; 2007.

32. Altamirano CHB. La Telesalud en Perú. Diagnóstico y Propuestas de Mejora. Gob gest pública. 2015; 2(1):53-73.

33. Ruiz EF, Proaño Á, Ponce OJ, Curioso WH. Tecnologías móviles para la salud pública en el Perú: lecciones aprendidas. Rev Peru Med Exp Salud Publica. 2015; 32:264-72.

34. Blaya JA, Shin SS, Yagui M, et al. Reducing communication delays and improving quality of care with a tuberculosis laboratory information system in resource poor environments: A cluster randomized controlled trial. PLOS ONE. 2014; 9(4):e90110.

35. Young SD, Cumberland WG, Nianogo R, Menacho LA, Galea JT, Coates T. The HOPE social media intervention for global HIV prevention in Peru: a cluster randomised controlled trial. Lancet HIV. 2015; 2(1):e27-e32.

36. Rubinstein A, Miranda JJ, Beratarrechea A, et al. Effectiveness of an mHealth intervention to improve the cardiometabolic profile of people with prehypertension in low-resource urban settings in Latin America: a randomised controlled trial. Lancet Diabetes \& Endocrinology. 2016; 4(1):52-63.

37. Diez-Canseco F, Toyama M, Ipince A, et al. Integration of a Technology-Based Mental Health Screening Program Into Routine Practices of Primary Health Care Services in Peru (The Allillanchu 
Project): Development and Implementation. Journal of medical Internet research. 2018; 20(3):e100.

38. Mueller JL, Lam CT, Dahl D, et al. Portable Pocket colposcopy performs comparably to standard-of-care clinical colposcopy using acetic acid and Lugol's iodine as contrast mediators: an investigational study in Peru. BJOG. 2018; 125(10):1321-9.
39. Martinez A, Villarroel V, Seoane J, del Pozo F. A study of a rural telemedicine system in the Amazon region of Peru. Journal of telemedicine and telecare. 2004; 10(4):219-25.

40. World Health Organization. Declaration of Astana. Geneva: World Health Organization/UNICEF; 2018.

Recibido: 24/07/2020

Aceptado: 22/09/2020 